\title{
Charge equilibration and potential steps in organic semiconductor multilayers
}

Geert Brocks, Deniz Cakir, Menno Bokdam, Michel P de Jong and Mats Fahlman

\section{Linköping University Post Print}

N.B.: When citing this work, cite the original article.

Original Publication:

Geert Brocks, Deniz Cakir, Menno Bokdam, Michel P de Jong and Mats Fahlman, Charge equilibration and potential steps in organic semiconductor multilayers, 2012, Organic electronics, (13), 10, 1793-1801.

http://dx.doi.org/10.1016/j.orgel.2012.05.041

Copyright: Elsevier

http://www.elsevier.com/

Postprint available at: Linköping University Electronic Press

http://urn.kb.se/resolve?urn=urn:nbn:se:liu:diva-85631 


\title{
Charge equilibration and potential steps in organic semiconductor multilayers
}

\author{
Geert Brocks $^{\mathrm{a}}$, Deniz Çakır ${ }^{\mathrm{a}}$, Menno Bokdam ${ }^{\mathrm{a}}$, Michel P. de Jong ${ }^{\mathrm{b}}$, Mats Fahlman ${ }^{\mathrm{c}}$ \\ ${ }^{a}$ Computational Materials Science, Faculty of Science and Technology and MESA+ Institute for Nanotechnology, University of Twente, \\ P.O. Box 217, 7500 AE Enschede, The Netherlands. \\ ${ }^{b}$ MESA+ Institute for Nanotechnology, University of Twente, P.O. Box 217, 7500 AE Enschede, The Netherlands. \\ ${ }^{c}$ Department of Physics, Chemistry and Biology, Linköping University, SE-581 83 Linköping, Sweden.
}

\begin{abstract}
Substantial potential steps $\sim 0.5 \mathrm{eV}$ are frequently observed in organic multilayers of donor and acceptor molecules. Often such potential steps depend on the order in which the individual layers are deposited, or on which substrate they are deposited. In this paper we outline a model for these potential steps, based upon integer charge transfer between donors and acceptors, charge equilibration across the multilayer, and simple electrostatics. Each donor, acceptor, or substrate material is characterized by a pinning level, and the potential profile can be deduced from the sequential order of the layers, and the differences between their pinning levels. For particular orderings we predict that intrinsic potential differencess lead to electric fields across individual layers, which may falsely be interpreted as band bending.
\end{abstract}

Keywords: organic semiconductor, donor acceptor, potential step, multilayer

\section{Introduction}

Organic electronic devices usually consist of several thin film layers with corresponding interfaces that facilitate different functionalities, such as charge injection, transport, or charge separation/recombination $[1,2]$. Consequently, a detailed understanding of interface properties, energy level alignment in particular, is critical for guiding the improvement of device performance. The energy level alignment at all-organic and hybrid organic-inorganic interfaces has been the subject of extensive research efforts in recent years [3-12].

The interaction between the materials at organic interfaces often is rather weak. It does not involve the formation of chemical bonds at the interface, which determines the band offsets at inorganic heterojunctions $[13,14]$. To describe the potential offsets observed at organic interfaces, an empirical model has been formulated, based on the transfer of (an integer number of) electrons across an interface $[7,15]$. Recent theoretical work has shown that the assumptions of such an integer electron transfer, combined with simple electrostatics at the interface, allows one to calculate the potential offset at an interface [16].

The potential profile of a multilayered structure is likewise determined by electron transfer between the different organic layers, and between the substrate and the organic overlayers [15]. Such a charge equilibration throughout the whole multilayer has been demonstrated in TTF / TCNQ stacks, for instance. The Fermi level of a TTF / TCNQ

\footnotetext{
${ }^{*}$ Corresponding author

Email address: g.brocks@tnw.utwente.nl (Geert Brocks)
}

stack deposited on a low work function substrate is pinned by the top layer, irrespective of whether the stack starts with a TTF or a TCNQ layer [17]. This occurs only if all acceptor layers (TCNQ) are in equilibrium with both the substrate and the donor layers (TTF). Charge equilibration across an organic multilayer stack has some remarkable consequences. For instance, the potential step observed at a particular organic heterojunction can apparently depend upon the sequence of the different organic layers in the multilayer [18].

In this paper we extend the integer charge transfer model to describe the potential profile in multilayer stacks containing organic-organic and/or metal-organic heterojunctions. The profile can be derived from the Fermi energy pinning levels of the individual organic donor and acceptor layers and the metal work functions. We find that the potential step at any heterojunction is fixed by the difference between the pinning levels or work functions left and right of the interface. In other words, the potential step is a property of the particular junction, and is independent of the other layers in the multilayer stack. However, charge equilibration within the stack can result in additional potential gradients across organic layers, which depend on the sequence of the layers in the stack.

Such a potential gradient has been found in TTF / TCNQ multilayers [17]. Our model shows that such gradients are quite general, and can be used to explain the apparent dependence of interface potential steps upon the layer sequence [18], or interpret cases where apparent "band bending" is observed [19].

This paper is organized as follows. In section 2 we explain our model for potential steps at interfaces and ac- 
cross organic multilayers. We find that trilayers consisting of one donor and two different acceptor layers, or one acceptor and two different donor layers, cover the possible distinct logical cases. Conceptually, a metallic substrate can play the role of either a donor or an acceptor layer. In section 3 we demonstrate the model on a number of examples, and section 4 contains the conclusions.

\section{Theory}

\subsection{Charge transfer across a single interface}

A simple model is presented describing the potential energy step at a single interface, caused by charge transfer between a layer of donor molecules (D) and a layer of acceptor molecules (A), see Fig. 1(a)-(c). We restrict ourselves to the case where the interactions between neutral molecules are small, and neglect the small induced dipoles caused by those intermolecular interactions [20, 21]. Likewise we neglect temperature effects [12].

We show that the potential step at a donor-acceptor interface can be interpreted as the difference between characteristic energy levels of the donor layer $W_{\mathrm{D}}^{+}$and acceptor layer $W_{\mathrm{A}}^{-}$, the so-called "pinning levels". A metal can act as either a donor or an acceptor layer. Potential energy steps at metal-donor or metal-acceptor interfaces can then be described within the same model, with the metal work function $W_{\mathrm{M}}$ playing the role of pinning level.

We consider a layer of acceptor molecules (A) stacked on top of a layer of donor molecules (D), Fig. 1(a)-(c), and assume that $N$ donor molecules have transferred one electron each to an acceptor molecule. The change in total energy with respect to the neutral case is

$$
E(N)=N\left(I_{\mathrm{D}}-A_{\mathrm{A}}\right)+E_{\mathrm{C}}(N),
$$

with $I_{\mathrm{D}}=E_{\mathrm{D}}^{+}-E_{\mathrm{D}}^{0}$ and $A_{\mathrm{A}}=E_{\mathrm{A}}^{0}-E_{\mathrm{A}}^{-}$the ionization potential of a donor molecule, and the electron affinity of an acceptor molecule, respectively. $E_{\mathrm{A}}^{0 /-}$ is the total energy of a neutral/negatively charged acceptor molecule and $E_{\mathrm{D}}^{0 /+}$ is the total energy of a neutral/positively charged donor molecule. $E_{\mathrm{C}}(N)$ is the electrostatic interaction energy between all charged molecules, polarization effects included.

A transfer of electrons between two layers results in a potential energy step along the direction normal to the layers. The simplest model for the electrostatics in a layered geometry is a parallel plate capacitor, which gives a potential energy step

$$
V=\frac{N e^{2}}{C},
$$

with $C$ the capacitance. It also suggests a simple model for the electrostatic energy

$$
E_{\mathrm{C}}(N)=\frac{1}{2} N V-N B_{\mathrm{D}}^{+}-N B_{\mathrm{A}}^{-},
$$

where the first term on the right-hand side is the energy required to charge a parallel plate capacitor. This is a

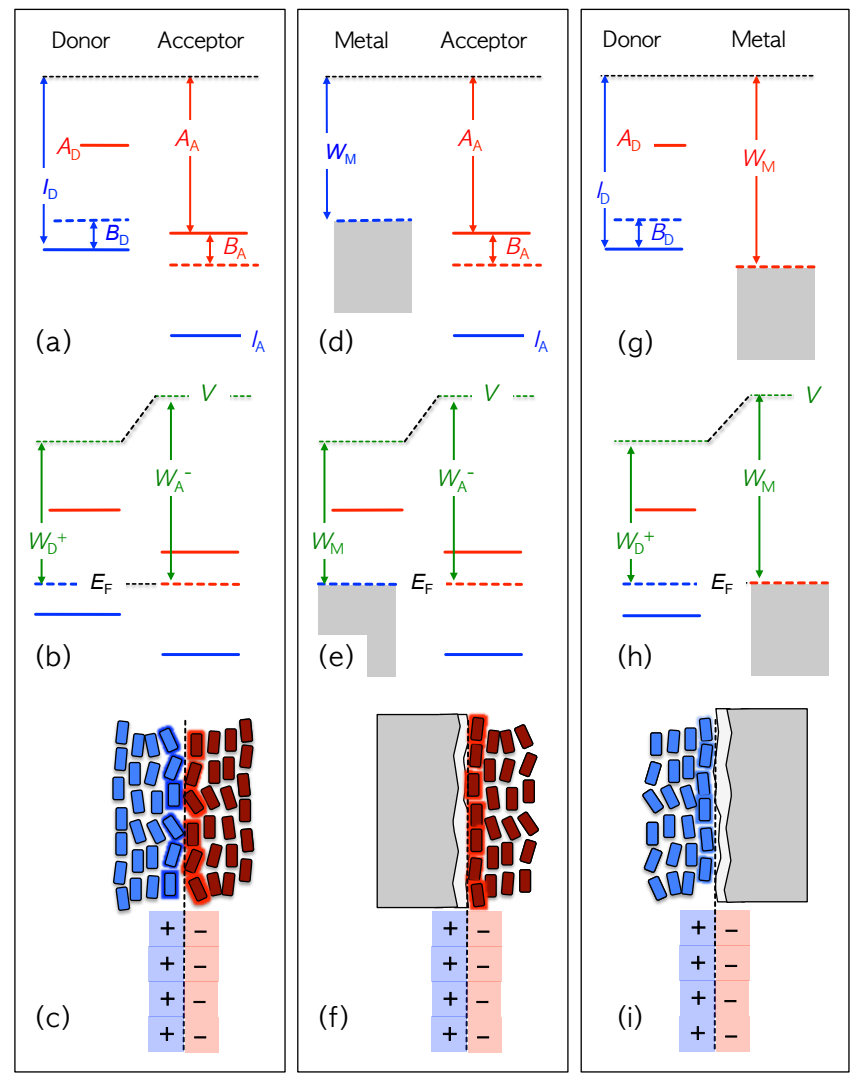

Figure 1: Energy diagrams for a bilayer of a donor and acceptor material, assuming $W_{\mathrm{A}}^{-}>W_{\mathrm{D}}^{+}$, before (a), and in equilibrium (b); schematic representation of the charge distribution (c). Same for a metal-acceptor interface, assuming $W_{\mathrm{A}}^{-}>W_{\mathrm{M}}$ (d)-(f), and for a donor-metal interface, assuming $W_{\mathrm{M}}>W_{\mathrm{D}}^{+}(\mathrm{g})-(\mathrm{i})$.

simple continuum model for the interface charging energy, which means that, besides containing the Coulomb interactions between the charged molecules, it also contains Coulomb interactions associated with charging individual donor and acceptor molecules. The latter are per definition accounted for in the ionization potential and the electron affinity of the molecules. To avoid double counting of these molecular charging energies, they have to be subtracted, if $E_{\mathrm{C}}$ is to represent the Coulomb interaction between the charged molecules only . The double counting correction is represented by the second and third terms on the right-hand side of Eq. (3)

It is in principle possible to calculate the charging energy $B_{\mathrm{D}}^{+}$or $B_{\mathrm{A}}^{-}$of an individual donor an acceptor molecule $[22,23]$, but this is not required here as we will show. Note however that the molecular charging energy generally decreases with the size of the molecule [22, 24]. More specifically, it decreases with increasing delocalization of the electrons or holes.

In equilibrium the total energy with respect to the number of charged molecules $E(N)$, Eq. (1), should be minimal, implying $d E / d N=0$, which determines the number of electrons transferred $N$. Using this in Eq. (2) then 
gives

$$
V=\left(A_{\mathrm{A}}+B_{\mathrm{A}}^{-}\right)-\left(I_{\mathrm{D}}-B_{\mathrm{D}}^{+}\right) .
$$

Note that we measure the potential energy step from the donor to the acceptor, which means $V \geq 0$, see Fig. 1(a)(c). If $A_{\mathrm{A}}+B_{\mathrm{A}}^{-}<I_{\mathrm{D}}-B_{\mathrm{D}}^{+}$, then no electrons are transferred, and $V=0$. In that case the vacuum levels left and right of the interface simply align.

The same model can be applied if the donor or the acceptor material is a metal. The ionization potential and the electron affinity are then given by the work function of the metal, $I=W_{\mathrm{M}}, A=W_{\mathrm{M}}$. Moreover, as the charge carriers in a metal are fully delocalized, $B^{+/-}=0$. The potential steps going from a metal to an acceptor material, respectively from a donor material to a metal, are given by

$$
\begin{aligned}
V & =\left(A_{\mathrm{A}}+B_{\mathrm{A}}^{-}\right)-W_{\mathrm{M}}, \\
& =W_{\mathrm{M}}-\left(I_{\mathrm{D}}-B_{\mathrm{D}}^{+}\right) .
\end{aligned}
$$

Again $V \geq 0$, as illustrated in Fig. 1(d)-(i).

If $V>0$, the work function on the acceptor side of a metal-acceptor interface is given by $W_{\mathrm{M}}+V=A_{\mathrm{A}}+B_{\mathrm{A}}^{-}$, see Fig. 1(d)-(f). The work function is then independent of the work function of the metal, or, in other words, the work function is pinned by the acceptor material. Likewise, if $V>0$, the work function on the donor side of a donor-metal interface $W_{\mathrm{M}}-V=I_{\mathrm{D}}-B_{\mathrm{D}}^{+}$is pinned by the donor material. Work function pinning levels for donor and acceptor materials are thus given by

$$
W_{\mathrm{D}}^{+}=I_{\mathrm{D}}-B_{\mathrm{D}}^{+} ; W_{\mathrm{A}}^{-}=A_{\mathrm{A}}+B_{\mathrm{A}}^{-} .
$$

Pinning levels can be obtained from experiment $[7,15]$, or from first-principles density functional theory calculations [16]. The ionization potential and electron affinitiy $I_{\mathrm{D}}$ and $A_{\mathrm{A}}$ of a molecule may depend on its local surroundings. The effect of polarization of the environment by a charged molecule drops out in the present model. Changing the polarization energy by $P$ leads to $I_{\mathrm{D}}^{\prime}=I_{\mathrm{D}}-P$ and $A_{\mathrm{A}}^{\prime}=A_{\mathrm{A}}+$ $P$. However, all energies associated with charging should be affected similarly, so $B_{\mathrm{D}}^{\prime}=B_{\mathrm{D}}^{+}-P$, $B_{\mathrm{A}}^{\prime-}=B_{\mathrm{A}}^{-}-P$. This implies that the pinning levels are not affected, i.e. $W_{\mathrm{D}}^{\prime+}=W_{\mathrm{D}}^{+}, W_{\mathrm{A}}^{\prime-}=W_{\mathrm{A}}^{-}$.

A similar argument cannot be constructed for the effects of the static electrostatic environment of a molecule. A change in the static environment can change the ionization potential considerably, as is demonstrated convincingly on molecular layers with different orientations [25]. The pinning level would be affected in a similar way. Therefore, molecular layers with different orientations should be treated as separate layers, each with its own pinning level.

A comparison to Eq. (4) shows that the potential step $V$ at a donor-acceptor interface is given by the difference in pinning levels $W_{\mathrm{A}}^{-}-W_{\mathrm{D}}^{+}$of the donor and the acceptor layers. Potential steps at interfaces with a metal are given by the differences between these pinning levels and the metal work function, Eq. (5), see Fig. 1.

\subsection{Charge equilibration in trilayers}

The model can be easily extended to describe multilayers. We will illustrate this on trilayers of donor and acceptor molecules. In a trilayer several different combinations and orderings of donor and acceptor layers are possible. Each possibility is discussed separately below. As discussed in the previous section, a metal can play the role of either a donor or an acceptor, so the results also hold for a stack of two different organic layers deposited onto a metal substrate.

We start with two layers of acceptor molecules $\left(A_{1}\right.$, $\mathrm{A}_{2}$ ) stacked on top of a layer of donor molecules (D), as shown in Fig. 2. Assume that the acceptor layers have received $N_{1}, N_{2}$ electrons, respectively. A straight-forward generalization of Eqs. (1) and (3) gives the total energy as

$$
\begin{aligned}
E\left(N_{1}, N_{2}\right) & =N_{1}\left(I_{\mathrm{D}}-A_{\mathrm{A}_{1}}\right) \\
& +N_{2}\left(I_{\mathrm{D}}-A_{\mathrm{A}_{2}}\right)+E_{\mathrm{C}}\left(N_{1}, N_{2}\right),
\end{aligned}
$$

and the electrostatic energy as

$$
\begin{aligned}
E_{\mathrm{C}}\left(N_{1}, N_{2}\right) & =\frac{1}{2} N_{1} V_{1}-N_{1} B_{\mathrm{D}}^{+}-N_{1} B_{\mathrm{A}_{1}}^{-} \\
& +\frac{1}{2} N_{2} V_{2}-N_{2} B_{\mathrm{D}}^{+}-N_{2} B_{\mathrm{A}_{2}}^{-} .
\end{aligned}
$$

Within a parallel plate capacitor model, the potentials $V_{1}, V_{2}$ of acceptor layers $\mathrm{A}_{1}, \mathrm{~A}_{2}$ are given by ${ }^{1}$

$$
V_{1}=\frac{\left(N_{1}+N_{2}\right) e^{2}}{C_{1}} ; V_{2}=\frac{N_{1} e^{2}}{C_{1}}+\frac{N_{2} e^{2}}{C_{2}} .
$$

Minimization of the total energy with respect to $N_{1}$ and $N_{2}$ then leads to simple expressions for the potential energy levels.

$$
V_{1}=W_{\mathrm{A}_{1}}^{-}-W_{\mathrm{D}}^{+} ; V_{2}=W_{\mathrm{A}_{2}}^{-}-W_{\mathrm{D}}^{+} .
$$

It means that, if the charge equilibrates across the trilayer, the potentials inside the different layers are determined by the pinning levels.

This derivation assumes that $V_{2} \geq V_{1} \geq 0$, implying $W_{\mathrm{A}_{2}}^{-} \geq W_{\mathrm{A}_{1}}^{-}$. This situation is shown in Fig. 2 (a)-(c). The potential step $V_{1}$ is between the donor and the acceptor layer $\mathrm{A}_{1}$. The increase of the potential from $V_{1}$ to $V_{2}$ occurs gradually over the acceptor layer $\mathrm{A}_{1}$. In other words, the potential step

$$
\Delta V=V_{2}-V_{1}=W_{\mathrm{A}_{2}}^{-}-W_{\mathrm{A}_{1}}^{-},
$$

is across the whole layer $A_{1}$, resulting in an intrinsic electric field across this layer. The field will drive negative

\footnotetext{
${ }^{1}$ For a stack of two dielectric layers of thickness $d_{1}$ and $d_{2}$, permittivities $\varepsilon_{1}$ and $\varepsilon_{2}$, and area $a$, the capacitances are $C_{1}=a \varepsilon_{1} / d_{1}$ and $C_{2}=a\left(d_{1} / \varepsilon_{1}+d_{2} / \varepsilon_{2}\right)^{-1}$. The precise form is not very important, as the potential energy levels do not depend on these capacitances.
} 


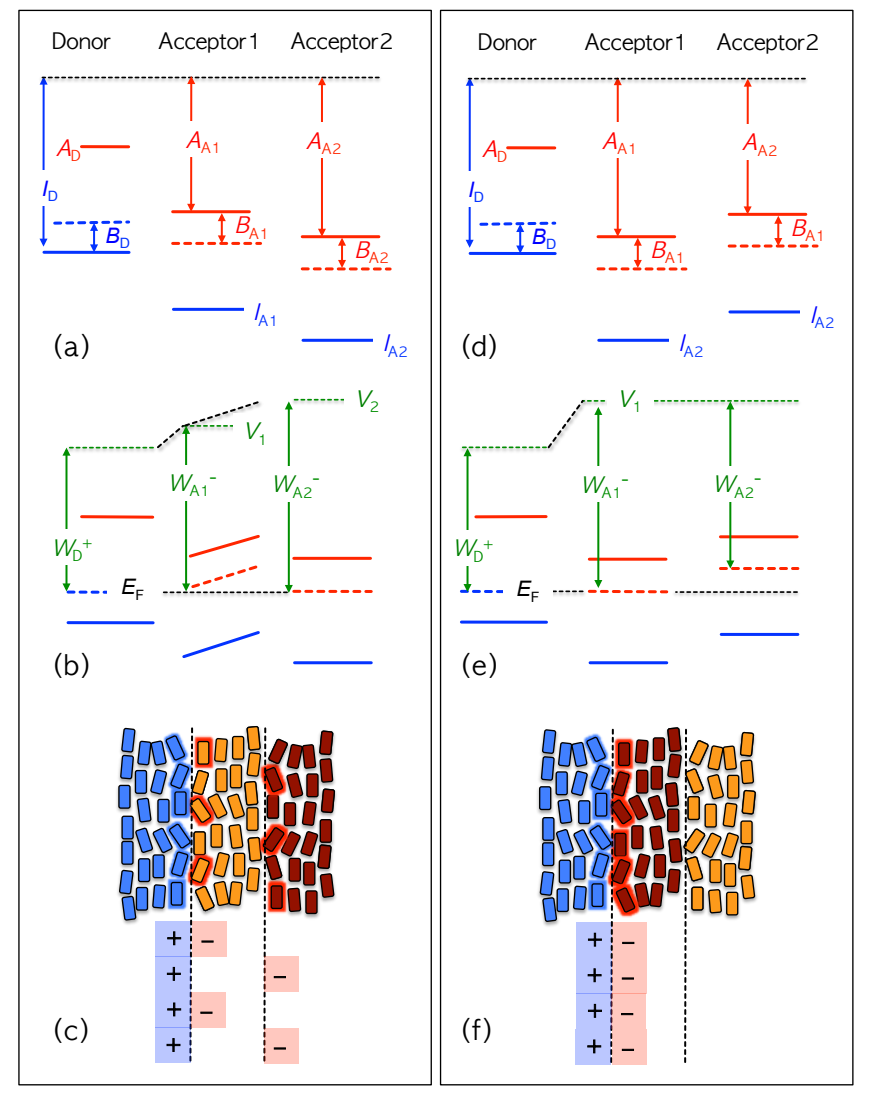

Figure 2: Energy diagrams for a trilayer of a donor and two acceptor materials, assuming $W_{\mathrm{A}_{2}}^{-}>W_{\mathrm{A}_{1}}^{-}$, before (a), and in equilibrium (b); schematic representation of the charge distribution (c). Same for $W_{\mathrm{A}_{2}}^{-}<W_{\mathrm{A}_{1}}^{-}(\mathrm{d})-(\mathrm{f})$.

charge carriers towards the donor layer $\mathrm{D}$ and positive charge carriers towards the second acceptor layer $\mathrm{A}_{2}$.

If $W_{\mathrm{A}_{2}}^{-}<W_{\mathrm{A}_{1}}^{-}$, then $V_{2}=V_{1}$, and $N_{2}=0$, i.e. there is no electron transfer to the acceptor layer $A_{2}$. This situation is shown in Fig. 2 (d)-(f). The electron transfer to layer $A_{1}$ shifts the electro-chemical potential to a value inside the gap of acceptor $\mathrm{A}_{2}$, below the pinning level of this acceptor. This renders the layer $\mathrm{A}_{2}$ inactive as an acceptor. Between layers $A_{1}$ and $A_{2}$ there is an alignment of the vacuum level.

We have also assumed that $W_{\mathrm{D}}^{+}<W_{\mathrm{A}_{1}}^{-}$and $W_{\mathrm{D}}^{+}<$ $W_{\mathrm{A}_{2}}^{-}$. If $W_{\mathrm{A}_{1}}^{-}<W_{\mathrm{D}}^{+}<W_{\mathrm{A}_{2}}^{-}$, then the acceptor layer $\mathrm{A}_{1}$ is not involved in any electron transfer, and acts as an insulator. The potential profile then looks like that of Fig. 2 (b), but without the step $V_{1}$ between donor D and acceptor $\mathrm{A}_{2}$, i.e. $V_{1}=0$. The potential increase to $V_{2}$ takes place across the whole layer $\mathrm{A}_{1}$ as before. If $W_{\mathrm{A}_{2}}^{-}<W_{\mathrm{D}}^{+}<W_{\mathrm{A}_{1}}^{-}$, then the acceptor $\mathrm{A}_{2}$ is inactive, and the potential profile is that shown in Fig. 2 (e). No charge carrier in layer $A_{1}$ would then experience a field-induced drift towards either interface in absence of an external bias, unlike the case illustrated in Fig. 2(a)-(c).

The special case $W_{\mathrm{A}_{2}}^{-}=W_{\mathrm{A}_{1}}^{-}$implies $V_{2}=V_{1}$, and $N_{2}=0$, i.e. there is no electron transfer to the acceptor

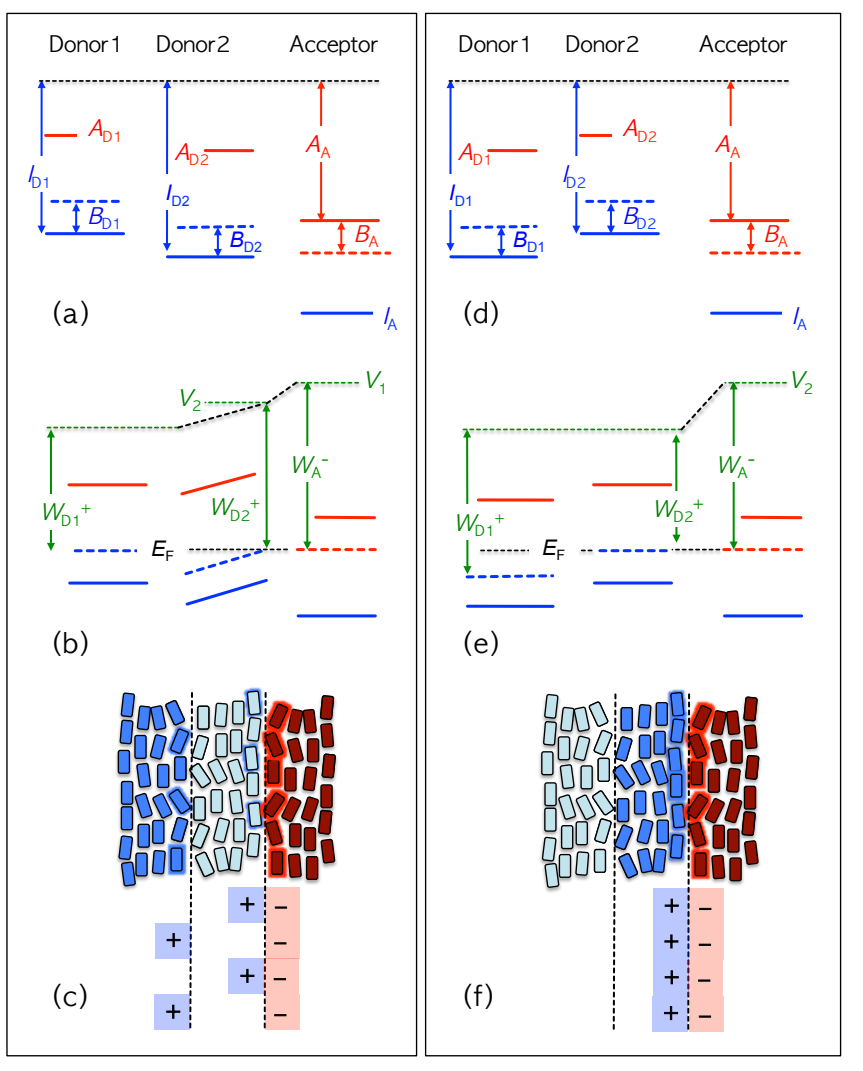

Figure 3: Energy diagrams for a trilayer of two donor and one acceptor material, assuming $W_{\mathrm{D}_{2}}^{+}>W_{\mathrm{D}_{1}}^{+}$, before (a), and in equilibrium (b); schematic representation of the charge distribution (c). Same for $W_{\mathrm{D}_{2}}^{+}<W_{\mathrm{D}_{1}}^{+}(\mathrm{d})-(\mathrm{f})$.

layer $\mathrm{A}_{2}$. This is obviously the case if the two acceptor layers are identical, $A_{1}=A_{2}$. The result implies that at a donor-acceptor interface with a homogeneous acceptor material, all transferred electrons tend to be as close to the interface as possible, i.e. within the molecular layers right at the interface.

The analysis works in a similar way for other types of multilayers. Consider one layer of acceptor molecules (A) stacked on top of two layers of donor molecules $\left(D_{1}, D_{2}\right)$, as shown in Fig. 3 (a)-(c). The potentials resulting from charge equilibration throughout the trilayer are given by

$$
V_{1}=W_{\mathrm{A}}^{-}-W_{\mathrm{D}_{1}}^{+} ; V_{2}=W_{\mathrm{D}_{2}}^{+}-W_{\mathrm{D}_{1}}^{+}
$$

This expression assumes that $V_{1} \geq V_{2} \geq 0$, implying $W_{\mathrm{D}_{1}}^{+} \leq W_{\mathrm{D}_{2}}^{+}$, as in Fig. 3 (a)-(c). A potential increase $V_{2}$ occurs over the whole donor layer $\mathrm{D}_{2}$, and between donor layer $\mathrm{D}_{2}$ and the acceptor layer there is a potential step

$$
\Delta V=V_{1}-V_{2}=W_{\mathrm{A}}^{-}-W_{\mathrm{D}_{2}}^{+} .
$$

The potential $V_{2}$ over donor layer $\mathrm{D}_{2}$ results in an electric field that will drive negative charge carriers to donor layer $\mathrm{D}_{1}$ and positive charge carriers to the acceptor layer $\mathrm{A}$.

If $W_{\mathrm{D}_{1}}^{+}>W_{\mathrm{D}_{2}}^{+}$, then the Fermi level is pinned by the equilibrium between donor layer $\mathrm{D}_{2}$ and the acceptor layer only, and there is no charge transfer to donor layer $\mathrm{D}_{1}$. 


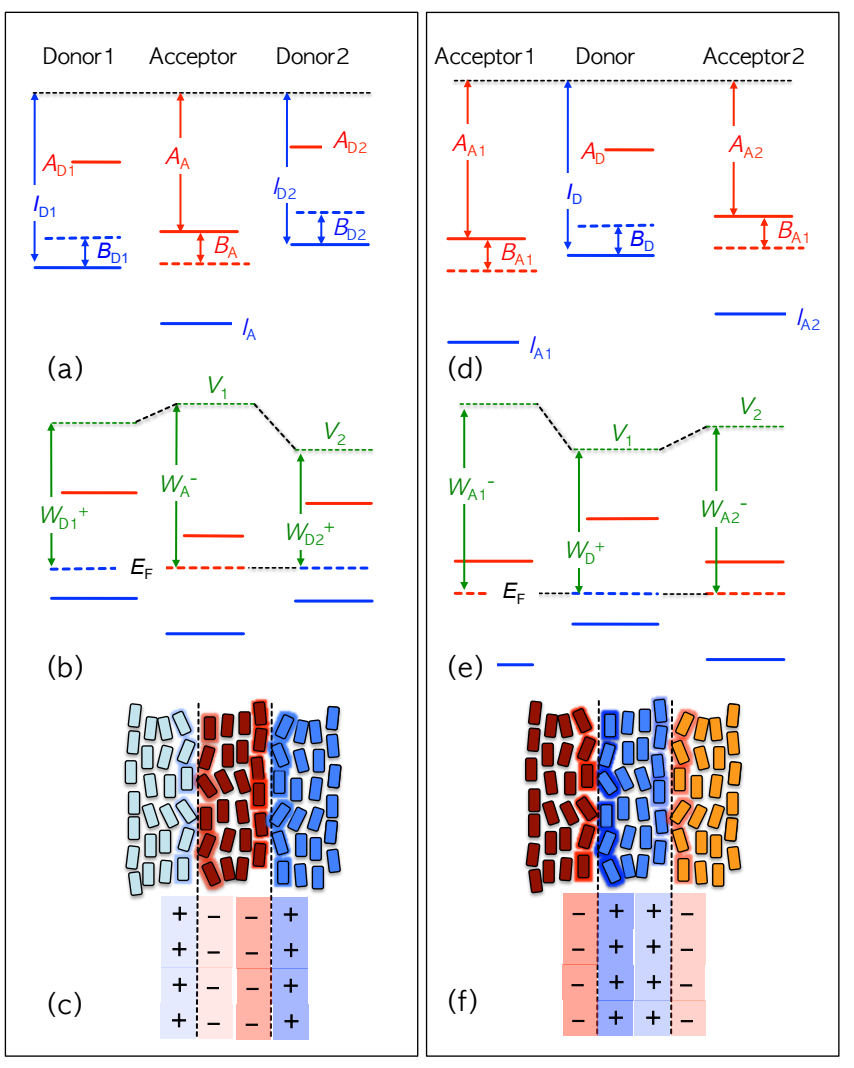

Figure 4: Energy diagrams for a trilayer of one acceptor material sandwiched between two donor materials, assuming $W_{\mathrm{A}}^{-}>W_{\mathrm{D}_{2}}^{+}$, $W_{\mathrm{D}_{1}}^{+}$, Eq. (4), before (a), and in equilibrium (b); schematic representation of the charge distribution (c).

Correspondingly, a potential step $V_{2}$ occurs between donor layer $\mathrm{D}_{2}$ and the acceptor layer, as Fig. 3 (d)-(f). There is no intrinsic electric field across donor layer $\mathrm{D}_{2}$ in contrast to the case shown in Fig. 3 (a)-(c).

We have assumed that $W_{\mathrm{D}_{1}}^{+}<W_{\mathrm{A}}^{-}$and $W_{\mathrm{D}_{2}}^{+}<W_{\mathrm{A}}^{-}$. If $W_{\mathrm{D}_{1}}^{+}<W_{\mathrm{A}}^{-}<W_{\mathrm{D}_{2}}^{+}$, then the donor layer $\mathrm{D}_{2}$ is inert. The potential profile is similar that of Fig. 3 (b), but without the step $V_{2}-V_{1}$ between donor $\mathrm{D}_{2}$ and acceptor A, i.e. $V_{2}=V_{1}$. The potential increase to $V_{1}$ takes place across the whole donor layer $\mathrm{D}_{2}$, which acts as an insulating layer. If $W_{\mathrm{D}_{2}}^{+}<W_{\mathrm{A}}^{-}<W_{\mathrm{D}_{1}}^{+}$, then the donor $\mathrm{D}_{1}$ is inactive, and the potential profile is as in Fig. 3 (e).

Another posibility is to have the acceptor layer sandwiched between the two donor layers $\mathrm{D}_{1}$ and $\mathrm{D}_{2}$, see Fig. 4(a) (c). Assuming that $W_{\mathrm{D}_{1}}^{+}<W_{\mathrm{A}}^{-}$and $W_{\mathrm{D}_{2}}^{+}<W_{\mathrm{A}}^{-}$, equilibrium throughout the trilayer results in the potential levels

$$
V_{1}=W_{\mathrm{A}}^{-}-W_{\mathrm{D}_{1}}^{+} ; V_{2}=W_{\mathrm{D}_{2}}^{+}-W_{\mathrm{D}_{1}}^{+} .
$$

A potential step $V_{1}$ is between the donor layer $\mathrm{D}_{1}$ and the acceptor layer A, and a second step $V_{2}-V_{1}=W_{\mathrm{D}_{2}}^{+}-W_{\mathrm{A}}^{-}$is between the acceptor layer and the donor layer $\mathrm{D}_{2}$. Note that $V_{1}>0$ and $V_{2}<V_{1}$. Finally there is the possibility of a donor layer sandwiched between two acceptor layers $A_{1}$ and $A_{2}$. This situation is shown in Fig. 4(d)-(f).

In all the cases we have considered so far, there is at

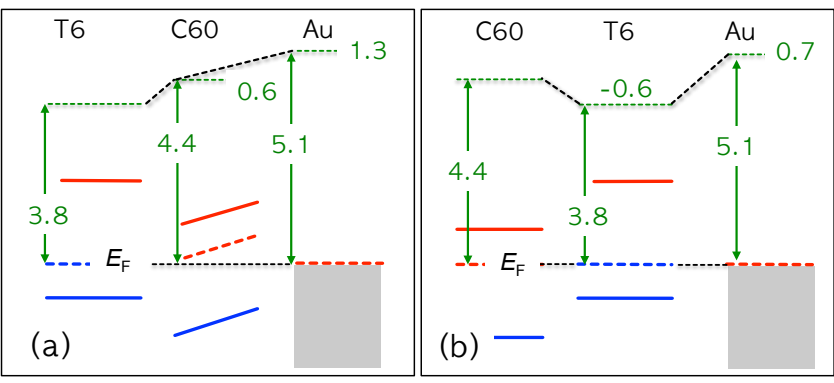

Figure 5: Energy diagrams for the trilayers T6 | C60 | Au (a), and $\mathrm{C} 60|\mathrm{~T} 6| \mathrm{Au}(\mathrm{b})$. The energies are in $\mathrm{eV}$.

least one combination for donor and acceptor materials for which $W_{\mathrm{D}}^{+}<W_{\mathrm{A}}^{-}$, so that a transfer of electrons between these materials takes place in order to establish equilibrium. If $W_{\mathrm{D}}^{+}>W_{\mathrm{A}}^{-}$for all combinations, then no charge transfer takes place. Consequently no potential steps are established and we have vacuum level alignment throughout the whole system.

These results also hold if one of the three materials is a metal electrode, replacing either the organic donor or the acceptor material. One obtains the relevant expressions by replacing the pinning level of either the donor $W_{\mathrm{D}}^{+}$or the acceptor $W_{\mathrm{A}}^{-}$in Eqs. (10), (12), or (14), by the work function of the metal $W_{\mathrm{M}}$. The donor/acceptor/metal combinations considered above enable one to work out the potential profile resulting from charge equilibration in any multilayer stack.

\section{Discussion}

We discuss a number of examples of multilayers and interpret the potential profiles observed in various experiments according to the model outlined in the previous section. The examples we pick, illustrate the different cases shown in Figs. 2-4. Table 1 summarizes the pinning levels and the work functions of the materials discussed in this paper. Pinning levels are obtained from first-principles calculations [16], or from experiment $[7,15]$. To establish a pinning level experimentally, one organic material is deposited on a range of metallic substrates with different work functions. Metal work functions are taken from experiment, as they very much depend on the particular surface conditions used.

Table 2 gives the potential levels $V_{1}$ and $V_{2}$ calculated for trilayers of these materials from the pinning levels and work functions of the individual materials. The agreement with the experimental levels obtained in measurements on trilayers is very good. In the following we will dicuss these trilayers one by one.

As a first example, in Ref. [26] layers of C60 and sexithiophene (T6) molecules are deposited on a Au substrate. $\mathrm{C} 60$ is an acceptor molecule and the calculated pinning 
Table 1: Summary of pinning levels and work functions (eV)

\begin{tabular}{|c|c|c|c|c|c|}
\hline Donor & $W_{\mathrm{D}}^{+}$ & Acceptor & $W_{\mathrm{A}}^{-}$ & Metal & $W_{\mathrm{M}}$ \\
\hline T6 & 3.8 & C60 & $4.4^{a}, 4.5^{b}$ & $\mathrm{AlO}_{x}$ & $3.6^{c}$ \\
\hline $\mathrm{TTF}$ & $4.2^{a, c}$ & TCNQ & $4.8^{c}$ & $\mathrm{Mg}$ & $3.7^{d}$ \\
\hline $\mathrm{CuPc}$ & $4.4^{a, e}$ & & & ITO & $5.0^{d}$ \\
\hline $\mathrm{MTD}^{h}$ & $4.5^{g}$ & & & $\mathrm{Au}$ & $5.1^{f}$ \\
\hline CBP & $5.2^{g}$ & & & $\mathrm{PED}^{i}$ & $5.7^{g}$ \\
\hline
\end{tabular}

Table 2: Potential levels $V_{1}$ and $V_{2}(\mathrm{eV})$ in trilayers calculated from pinning levels. The numbers between ( ) give the measured values. The column marked "fig" refers to the different cases represented by the figures.

\begin{tabular}{lccl}
\hline Stack & $V_{1}$ & $V_{2}$ & Fig \\
\hline $\mathrm{T} 6 / \mathrm{C} 60 / \mathrm{Au}$ & $0.6\left(0.6^{a}\right)$ & $1.3\left(1.2^{a}\right)$ & 2a-c, 5a \\
$\mathrm{C} 60 / \mathrm{T} 6 / \mathrm{Au}$ & $-0.6\left(-0.6^{a}\right)$ & $0.7\left(0.6^{a}\right)$ & $4 \mathrm{~d}-\mathrm{f}, 5 \mathrm{~b}$ \\
$\mathrm{AlO}_{x} / \mathrm{TCNQ} / \mathrm{TTF}$ & $1.2\left(1.2^{b}\right)$ & $0.6\left(0.6^{b}\right)$ & $4 \mathrm{a}-\mathrm{c}, 6 \mathrm{a}$ \\
$\mathrm{AlO}_{x} / \mathrm{TTF} / \mathrm{TCNQ}$ & $1.2\left(1.2^{b}\right)$ & $0.6\left(0.6^{b}\right)$ & $3 \mathrm{a}-\mathrm{c}, 6 \mathrm{~b}$ \\
$\mathrm{MTD} / \mathrm{CBP} / \mathrm{PED}$ & $1.2\left(1.1^{c}\right)$ & $0.7\left(0.7^{c}\right)$ & $3 \mathrm{a}-\mathrm{c}, 7 \mathrm{a}$ \\
$\mathrm{CBP} / \mathrm{MTD} / \mathrm{PED}$ & $0\left(0.1^{c}\right)$ & $1.2\left(1.3^{c}\right)$ & $3 \mathrm{~d}-\mathrm{f}, 7 \mathrm{~b}$ \\
$\mathrm{Mg} / \mathrm{CuPc} / \mathrm{C} 60$ & $0.8\left(0.8^{d}\right)$ & $0.7\left(0.7^{d}\right)$ & $3 \mathrm{a}-\mathrm{c}, 8 \mathrm{a}$ \\
ITO$/ \mathrm{CuPc} / \mathrm{C} 60^{\text {Refs. }}{ }^{a}[26] ;{ }^{b}\left[17{ }^{c}{ }^{c}[18] ;{ }^{d}[19]\right.$ & &
\end{tabular}

level of a $\mathrm{C} 60$ layer is $W_{\mathrm{C} 60}^{-}=4.4 \mathrm{eV}$ [16]. The calculated pinning level of a (standing) layer of the donor molecule T6 is $W_{\mathrm{T} 6}^{+}=3.8 \mathrm{eV}$. The $\mathrm{Au}$ substrate has a measured work function $W_{\mathrm{Au}}=5.1 \mathrm{eV}[26]$. Therefore, both $\mathrm{Au}$ and C60 act as an acceptor with respect to T6. The trilayer T6 | C60 | Au then corresponds to the Donor | Acceptor1 | Acceptor2 stack shown in Fig. 2 (b), with $W_{\mathrm{D}}^{+}=W_{\mathrm{T} 6}^{+}$, $W_{\mathrm{A} 1}^{-}=W_{\mathrm{C} 60}^{-}$, and $W_{\mathrm{A} 2}^{-}=W_{\mathrm{Au}}$. The calculated potential levels are $V_{1}=4.4-3.8=0.6 \mathrm{eV}$ and $V_{2}=5.1-3.8=1.3$ $\mathrm{eV}$. The results are shown in Fig. 5(a).

These numbers are in good agreement with the potential levels observed in experiment [26]. Note however that, whereas the potential step $V_{1}$ is between the T6 and the C60 layers, the further increase to $V_{2}$ in our model takes place across the whole C60 layer. This means that over the thickness $d$ of the C60 layer there should be an average electric field of $\left(V_{2}-V_{1}\right) / d$.

Interchanging the order of deposition of T6 and C60 layers onto the $\mathrm{Au}$ substrate, one obtains the trilayer C60 | T6 | Au. This corresponds to the Acceptor1 | Donor | Acceptor 2 case shown in Fig. 4 (e). Between the C60 and the $\mathrm{T} 6$ layer there is a potential step $V_{1}=3.8-4.4=$ $-0.6 \mathrm{eV}$. The potential level of $\mathrm{Au}$ with respect to $\mathrm{C} 60$ is $V_{2}=5.1-4.4=0.7 \mathrm{eV}$, which leads to a potential step $V_{2}-V_{1}=1.3 \mathrm{eV}$ between the $\mathrm{T} 6$ layer and the $\mathrm{Au}$ substrate. This situation is shown in Fig. 5(b). Again the numbers are in good agreement with experiment [26]. Note that in contrast to the case discussed above, the potential steps are now between the layers, i.e. there are no intrinsic electric fields across the layers.

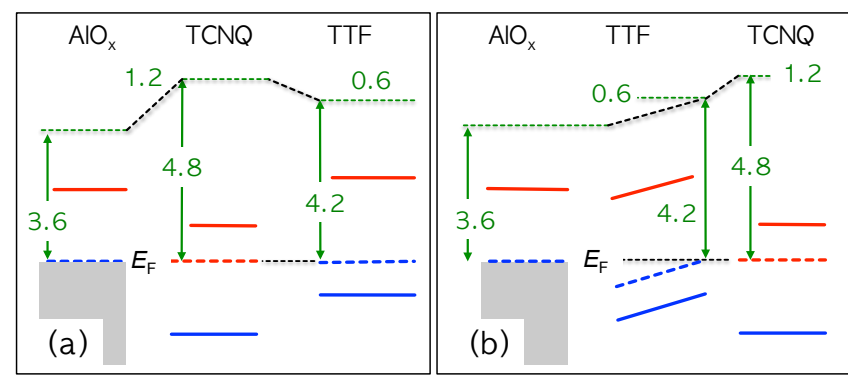

Figure 6: Energy diagrams for the trilayers $\mathrm{AlO}_{x}|\mathrm{TCNQ}| \mathrm{TTF}$ (a), and $\mathrm{AlO}_{x}|\mathrm{TTF}| \mathrm{TCNQ}$ (b). The energies are in $\mathrm{eV}$.

In Ref. [17] a stack of layers of the donor molecule TTF and layers of the acceptor molecule TCNQ is deposited onto a partially oxidized Al surface. TTF and TCNQ have experimental pinning levels $W_{\mathrm{TTF}}^{+}=4.2 \mathrm{eV}$, and $W_{\mathrm{TCNQ}}^{-}=4.8 \mathrm{eV}$, respectively [27]. As the $\mathrm{AlO}_{x}$ electrode has a work function $W_{\mathrm{Al}}=3.6 \mathrm{eV}$, it should therefore act as a donor with respect to TCNQ. The trilayer $\mathrm{AlO}_{x}|\mathrm{TCNQ}| \mathrm{TTF}$ then acts as the Donor1 | Acceptor | Donor2 stack shown in Fig. 4 (b), with $W_{\mathrm{D} 1}^{+}=W_{\mathrm{AlO}}$, $W_{\mathrm{A}}^{-}=W_{\mathrm{TCNQ}}^{-}$, and $W_{\mathrm{D} 2}^{+}=W_{\mathrm{TTF}}^{+}$. The corresponding potential levels are $V_{1}=4.8-3.6=1.2 \mathrm{eV}$, and $V_{2}=4.2-3.6=0.6 \mathrm{eV}$. The result is shown in Fig. 6 (a).

If the TCNQ and the TTF layers are interchanged to give the trilayer $\mathrm{AlO}_{x}|\mathrm{TTF}| \mathrm{TCNQ}$, one obtains the Donor1 | Donor2 | Acceptor case shown in Fig. 3 (b). Across the whole TTF layer there is a potential step $V_{2}=$ $4.2-3.6=0.6 \mathrm{eV}$. The potential level $V_{1}=4.8-3.6=1.2$ $\mathrm{eV}$, implying that between the TTF and the TCNQ layer there is a potential step $V_{1}-V_{2}=1.2-0.6=0.6 \mathrm{eV}$. This situation is shown in Fig. 6 (b). This analysis agrees with that given in Ref. [17].

A further example comes from Ref. [18], where layers of CBP and $m$-MTDATA molecules are deposited onto a PEDOT-PFESA substrate. A CBP layer has an experimental pinning level $W_{\mathrm{CBP}}^{+}=5.2 \mathrm{eV}$, and a $m$-MTDATA layer a pinning level $W_{\text {MTDATA }}^{+}=4.5 \mathrm{eV}$ [28]. The PEDOTPFESA electrode has a work function $W_{\text {PEDOT }}=5.7 \mathrm{eV}$, implying that it should act as an acceptor with respect to both the CBP molecules, as well as the $m$-MTDATA molecules. The trilayer MTDATA | CBP | PEDOT then corresponds to the Donor1 | Donor2 | Acceptor stack shown in Fig. 3 (b), with $W_{\mathrm{D} 1}^{+}=W_{\mathrm{MTDATA}}^{+}, W_{\mathrm{D} 2}^{+}=W_{\mathrm{CBP}}^{+}$, and $W_{\mathrm{A}}^{-}=W_{\text {PEDOT }}$. It leads to the potential levels $V_{2}=5.2-4.5=0.7 \mathrm{eV}$, and $V_{1}=5.7-4.5=1.2 \mathrm{eV}$, as illustrated in Fig. 7 (a). A potential step $V_{1}-V_{2}=0.5$ $\mathrm{eV}$ occurs between the CBP and the PEDOT layer. These numbers agree with the experimental observations [18]. Again our model predicts that the potential step $V_{2}$ takes place across the entire CBP layer.

Interchanging the $m$-MTDATA and CBP layers leads to the trilayer CBP | MTDATA | PEDOT, and to the Donor1 | Donor2 | Acceptor situation shown in Fig. 3 (e). 


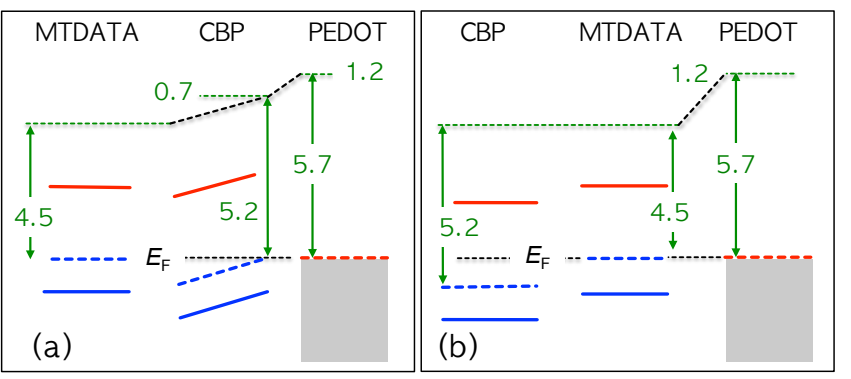

Figure 7: Energy diagrams for the trilayers MTDATA | CBP | PEDOT (a), and CBP | MTDATA | PEDOT (b). The energies are in $\mathrm{eV}$.

In this case, a large potential step $V_{1}=5.7-4.5=1.2$ $\mathrm{eV}$ occurs between the $m$-MTDATA and the PEDOT layers. There is a line-up of the vacuum levels between the $m$-MTDATA and the CBP layers, corresponding to an absense of charge transfer to the CBP layer, as shown in Fig. 7 (b).

Finally, in Ref. [19] layers of CuPc and C60 are deposited onto a $\mathrm{Mg}$ substrate and onto a ITO substrate. $\mathrm{CuPc}$ and $\mathrm{C} 60$ layers have experimental pinning levels $W_{\mathrm{CuPc}}^{+}=4.4 \mathrm{eV}$ and $W_{\mathrm{C} 60}^{-}=4.5 \mathrm{eV}[29,30] . \mathrm{Mg}$ and ITO have work functions $W_{\mathrm{Mg}}=3.7 \mathrm{eV}$, and $W_{\mathrm{ITO}}=5.0$ $\mathrm{eV}$, respectively. It means that $\mathrm{Mg}$ acts as donor with respect to the C60 layer, and ITO acts as acceptor with respect to the $\mathrm{CuPc}$ layer. The stack $\mathrm{Mg}|\mathrm{CuPc}| \mathrm{C} 60$ then correponds to the Donor1 | Donor2 $\mid$ Acceptor situation as shown in Fig. 3 (b), with $W_{\mathrm{D} 1}^{+}=W_{\mathrm{Mg}}, W_{\mathrm{D} 2}^{+}=W_{\mathrm{CuPc}}^{+}$, and $W_{\mathrm{A}}^{-}=W_{\mathrm{C} 60}^{-}$. The corresponding potential levels are $V_{1}=4.4-3.7=0.7 \mathrm{eV}$, and $V_{2}=4.5-3.7=0.8 \mathrm{eV}$, as shown in Fig. 8 (a). Note that also in this case we predict the potential step $V_{1}$ to occur across the entire CuPc layer. The electric field resulting from this potential step may be a source of the "band bending" observed in Ref. [19].

The stack ITO | CuPc | C60 corresponds to the Acceptor1 | Donor | Acceptor2 situation shown in Fig. 4 (e), with $W_{\mathrm{A} 1}^{-}=W_{\mathrm{ITO}}, W_{\mathrm{D}}^{+}=W_{\mathrm{CuPc}}^{+}$, and $W_{\mathrm{A} 2}^{-}=W_{\mathrm{C} 60}^{-}$. The corresponding potential levels are $V_{1}=4.4-5.0=-0.6$ $\mathrm{eV}$, and $V_{2}=4.5-5.0=-0.5 \mathrm{eV}$. In this case we predict that the potential step $V_{1}$ occurs between the ITO and the $\mathrm{CuPc}$ layers. The results are shown in Fig. 8 (b). The experimentally observed potential steps agree with this analysis [19].

\section{Conclusions}

In conclusion, we have developed a model to describe potential steps occuring in organic multilayers of donor and acceptor molecules. The model is based upon the assumptions of integer charge transfer between donor and acceptor layers, charge equilibration across the multilayer, and simple electrostatics. Each donor or acceptor material is characterized by a pinning level, which can be obtained from first-principles calculations, or from experiment. A

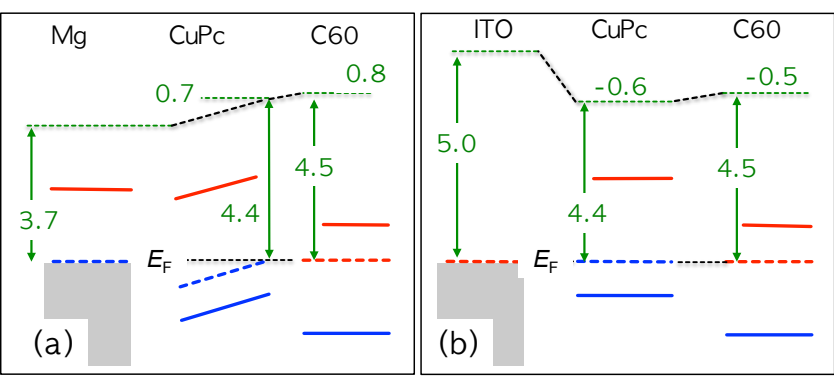

Figure 8: Energy diagrams for the trilayers $\mathrm{Mg}|\mathrm{CuPc}| \mathrm{C} 60$ (a), and ITO $|\mathrm{CuPc}| \mathrm{C} 60$ (b). The energies are in $\mathrm{eV}$.

metallic electrode can replace either a donor, or an acceptor layer within the model, its work function playing the role of the pinning level.

The potential profile in a multilayer can be deduced from the ordering of the layers, and their individual pinning levels. Electron transfer at a donor $\mid$ acceptor interface results in a potential step equal to the difference between the donor and acceptor pinning levels. This potential step is localized at the interface, and is a property of that particular interface. A sequence of a donor layer and two different acceptor layers, or of two different donor layers and an acceptor layer, can result in a potential gradient across the middle layer. This correponds to an electric field across this layer, which might be falsely interpreted as "band bending". The model is tested on a range of examples.

\section{Acknowledgment}

This work is part of the European project MINOTOR, grant no. FP7-NMP-228424. MF acknowledges support from STEM, the Swedish Energy Agency.

[1] C. W. Tang, Appl. Phys. Lett. 48 (1986) 183-185.

[2] C. W. Tang, S. A. VanSlyke, Appl. Phys. Lett. 51 (1987) 913915.

[3] H. Ishii, K. Sugiyama, E. Ito, K. Seki, Adv. Mater. 11 (1999) 605-625.

[4] A. Kahn, N. Koch, W. Gao, Journal of Polymer Science: Part B: Polymer Physics 41 (2003) 2529-2548.

[5] H. Vázquez, W. Gao, F. Flores, A. Kahn, Phys. Rev. B 71 (2005) 041306(R).

[6] C. Tengstedt, W. Osikowicz, W. R. Salaneck, I. D. Parker, C. H. Hsu, M. Fahlman, Appl. Phys. Lett. 88 (2006) 053502.

[7] M. Fahlman, A. Crispin, X. Crispin, S. K. M. Henze, M. P. de Jong, W. Osikowicz, C. Tengstedt, W. R. Salaneck, J. Phys.: Condens. Matter 19 (2007) 183202.

[8] H. Fukagawa, S. Kera, T. Kataoka, S. Hosoumi, Y. Watanabe, K. Kudo, N. Ueno, Adv. Mater. 19 (2007) 665-668.

[9] H. Vázquez, F. Flores, A. Kahn, Org. Elec. 8 (2007) 241-248.

[10] N. Koch, J. Phys.: Condens. Matter 20 (2008) 184008.

[11] W. Chen, D. C. Qi, Y. L. Huang, H. Huang, Y. Z. Wang, S. Chen, X. Y. Gao, A. T. S. Wee, J. Phys. Chem. C 113 (2009) 12832-12839.

[12] M. T. Greiner, M. G. Helander, W. M. Tang, Z. B. Wang, J. Qiu, Z. H. Lu, Nat. Mater. 11 (2011) 76-81.

[13] A. Franciosi, C. G. van de Walle, Surf. Sci. Rep. 25 (1996) 1140.

[14] J. Tersoff, Phys. Rev. B 30 (1984) 4874-4877(R). 
[15] S. Braun, W. R. Salaneck, M. Fahlman, Adv. Mater. 21 (2009) 1450-1472.

[16] M. Bokdam, D. Çakır, G. Brocks, Appl. Phys. Lett. 98 (2011) 113303.

[17] S. Braun, X. Liu, W. R. Salaneck, M. Fahlman, Org. Elec. 11 (2010) 212-217.

[18] S. Braun, M. P. de Jong, W. Osikowicz, W. R. Salaneck, Appl. Phys. Lett. 91 (2007) 202108.

[19] Y. C. Zhou, Z. T. Liu, J. X. Tang, C. S. Lee, S. T. Lee, J. El. Spectrosc. 174 (2009) 35-39.

[20] M. Linares, D. Beljonne, J. Cornil, K. Lancaster, J.-L. Brédas, S. Verlaak, A. Mityashin, P. Heremans, A. Fuchs, C. Lennartz, J. Idé, R. Mereau, P. Aurel, L. Ducasse, F. Castet, J. Phys. Chem. C 114 (2010) 3215-3224.

[21] M. Bokdam, P. A. Khomyakov, G. Brocks, Z. Zhong, P. J. Kelly, Nano Lett. 11 (2011) 4631-4635.

[22] G. Brocks, J. van den Brink, A. F. Morpurgo, Phys. Rev. Lett. 93 (2004) 146405.

[23] G. Giovannetti, G. Brocks, J. van den Brink, Phys. Rev. B 77 (2008) 035133.

[24] G. Brocks, Synth. Met. 102 (1999) 914-915.

[25] S. Duhm, G. Heimel, I. Salzmann, H. Glowatzki, R. L. Johnson, A. Vollmer, J. Rabe, N. Koch, Nature Mater. 7 (2008) 326-332.

[26] Y. Ge, J. E. Whitten, Chem. Phys. Lett. 448 (2007) 65-69.

[27] R. J. Murdey, W. R. Salaneck, Jpn. J. Appl. Phys. Part 144 (2005) 3751-3756.

[28] S. Braun, W. Osikowicz, Y. Wang, W. R. Salaneck, Org. Elec. 8 (2007) 14-20.

[29] H. Peisert, A. Petr, L. Dunsch, T. Chassé, M. Knupfer, ChemPhysChem 8 (2007) 386-390.

[30] W. Osikowicz, M. P. de Jong, W. R. Salaneck, Adv. Mater. 19 (2007) 4213-4217. 